\title{
La revolución molecular del cáncer de pulmón en el siglo XXI
}

\author{
Pedro Ramos
}

a incidencia mundial de cáncer de pulmón en 2018, según la información de Globocan, es de 2.093 .876 (11,6\%) casos nuevos y causa $1.761 .007(18,4 \%)$ muertes, ocupando el primer lugar en incidencia y mortalidad1.

En Colombia es la quinta causa de incidencia, con $5.856(5,7 \%)$ casos nuevos, y causa $5.236(12,43 \%)$ muertes, siendo la segunda causa de mortalidad por cáncer ${ }^{1}$.

Con la publicación en 1995 del metaanálisis en BMJ, se demostró el beneficio de la quimioterapia sobre el mejor cuidado de soporte con efecto en mejoría en la sobrevida a un año del 15\% versus el 5\%, respectivamente 2 .

Múltiples estudios posteriores que han comparado regímenes de quimioterapia que se usan para mantenimiento y adicionan antiangiogénicos han reportado mejoría en la sobrevida global ${ }^{3}$.

Uno de los mayores avances en el tratamiento de cáncer de pulmón de células no pequeñas (NSCLC) ha sido la identificación de mutaciones driver, que dirigen el desarrollo y progresión del cáncer de pulmón, y son blanco terapéutico; las investigaciones han señalado mejoría sustancial en la sobrevida libre de progresión, aunque no en la sobrevida global, por el efecto crossover ${ }^{4}$.

Los medicamentos disponibles para terapia blanco en casos de mutación EGFR son osimertinib, erlotinib, gefitinib y afatinib en primera línea, con alternativas de combinaciones de erlotinib con bevacizumab, gefitinib más quimioterapia y otros antiangiogénicos en combinación con EGFR-ITK, los cuales han demostrado mejoría en la sobrevida ${ }^{4,5}$.

Para otros tipos de mutaciones driver, como ALK, ROSI-1, BRAF, HER2, MET, RET y NTRK, disponemos de tratamientos blanco, algunos con aprobación y uso rutinario en la práctica clínica ${ }^{6}$.

El siguiente gran avance fue el desarrollo de los inmune check-point inhibitors, anticuerpos que tienen blanco en la vía de muerte programada (PD-1 y su ligando PD-L1) y son efectivos en varios tipos de cáncer. Tenemos disponible el pembrolizumab para uso en tratamiento de primera línea de cáncer de pulmón de células no pequeñas en pacientes con PD-L1, expresión de células tumorales del 50\% o más, con disminución de riesgo de muerte hasta del $40 \%$.

Otros análisis han explorado en primera línea nivolumab más ipilimumab, usando como biomarcador predictivo la carga mutacional del tumor (TMB) mayor de 10, que demostró impacto en la sobrevida, con el inconveniente de que este biomarcador no es de uso rutinario en la práctica clínica .

Otro inhibidor anti-PD-L1 es el atezolimumab, el cual se ha estudiado en combinación con quimioterapia, con o sin antiangiogénico, que es otra opción en primera línea que se ha evaluado en varios estudios (IMpower 150, 130 y 132) con impacto en la sobrevida y aprobación por autoridades regulatorias ${ }^{9-11}$.

El durvalumab más tremelimumab en pacientes con NSCLC metastásicos y con TMB mayor de 20 mejora la sobrevida, pero se requieren datos adicionales antes de su uso práctico rutinario; sin embargo, sí está aprobado en pacientes en estadio III que no progresen a la combinación quimioterapia y radioterapia como mantenimiento ${ }^{12,13}$.

El estudio de Gandy y colaboradores en pacientes con NSCLC avanzado sin mutación driver estableció el estándar de quimioterapia más pembrolizumab en pacientes con PD-L1 del 1-49\% y menor al 1\%, con impacto en sobrevida a un año del $69 \%$ y reducción del riesgo de muerte del $51 \%{ }^{14}$

Hay muchas dudas acerca de cuál es el biomarcador predictivo de beneficios de los inhibidores check-point. El PD-L1 es problemático en algunos estudios, pues su beneficio se da si es solo del 50\% o más; en otros hay beneficio hasta en menos del 1\%. EL TMB, otro biomarcador de uso en ensayos clínicos, que no se ha incorporado a la práctica clínica rutinaria, está en investigación activa para determinar la combinación de biomarcadores más predictiva de resultados ${ }^{15}$.

Finalmente, se puede concluir que hay un avance creciente y sustancial en el tratamiento del cáncer de pulmón de células no pequeñas que ha permitido mejorar la sobrevida a largo plazo de estos pacientes, cuyo pronóstico era mucho más adverso en décadas anteriores. 


\section{Referencias}

1. International Agency for Research on Cancer. Globocan 2018 [internet]. [citado 2018 Sep 15]. Disponible en: http://globocan.iarc.fr/

2. Non-small Cell Lung Cancer Collaborative Group. Chemotherapy in non-small cell lung cancer: a meta-analysis using updated data on individual patients from 52 randomised clinical trials. BMJ. 1995;311(7010):899-909.

3. Ciuleanu T, Brodowicz T, Zielinski C, Kim JH, Krzakowski M, Laack E, et al. Maintenance pemetrexed plus best supportive care versus placebo plus best supportive care for non-small-cell lung cancer: a randomised, double-blind, phase 3 study. Lancet. 2009;374(9699):1432-40.

4. Lee CK, Brown C, Gralla RJ, Hirsh V, Thongprasert S, Tsai CM, et al. Impact of EGFR inhibitor in non-small cell lung cancer on progression-free and overall survival: a meta-analysis. J Natl Cancer Inst. 2013;105(9):595-605.

5. Nakamura A, Inoue $A$, Morita $S$, et al. Phase III study comparing gefitinib monotherapy $(G)$ to combination therapy with gefitinib, carboplatin, and pemetrexed (GCP) for untreated patients (pts) with advanced non-small cell lung cancer (NSCLC) with EGFR mutations (NEJ009). J Clin Oncol. 2018;36S:ASCO \# 9005.

6. Lindeman $\mathrm{NI}$, Cagle PT, Aisner DL, Arcila ME, Beasley MB, Bernicker $\mathrm{EH}$, et al. Updated molecular testing guideline for the selection of lung cancer patients for treatment with targeted tyrosine kinase inhibitors: guideline from the College of American Pathologists, the International Association for the Study of Lung Cancer, and the Association for Molecular Pathology. J Mol Diagn. 2018;20(2):129-159.

7. Reck M, Rodríguez-Abreu $D$, Robinson AG, Hui R, Csőszi T, Fülöp $A$, et al. Pembrolizumab versus chemotherapy for PD-L1-positive nonsmall-cell lung cancer. N Engl J Med. 2016;375(19):1823-33.

8. Hellmann MD, Ciuleanu TE, Pluzanski A, Lee JS, Otterson GA Audigier-Valette $C$, et al. Nivolumab plus ipilimumab in lung cancer with a high tumor mutational burden. N Engl J Med. 2018;378(22):2093-2104.
9. Reck M, Mok TSK, Nishio M, Jotte RM, Cappuzzo F, Orlandi F, et al. Atezolizumab plus bevacizumab and chemotherapy in non-small-cell lung cancer (IMpower150): key subgroup analyses of patients with EGFR mutations or baseline liver metastases in a randomised, open-label phase 3 trial. Lancet Respir Med. 2019;7(5):387-401.

10. West $H$, McCleod M, Hussein M, Morabito A, Rittmeyer A, Conter $\mathrm{HJ}$, et al. Atezolizumab in combination with carboplatin plus nab-paclitaxel chemotherapy compared with chemotherapy alone as first-line treatment for metastatic non-squamous non-small-cell lung cancer (IMpower130): a multicentre, randomised, open-label, phase 3 trial. Lancet Oncol. 2019;20(7):924-37.

11. Papadimitrakopoulou VA, Cobo M, Bordoni $R$, et al. OA05.07 Mpower132: PFS and safety results with $1 \mathrm{~L}$ atezolizumab + carboplatin/cisplatin + pemetrexed in stage IV non-squamous NSCLC. J Thorac Oncol. 2018;13S:WCLC \# OA05.07.

12. Peters $S, C$ ho $B C$, Reinmuth $N$, et al. Tumor mutational burden (TMB) as a biomarker of survival in metastatic non-small cell lung cancer (mNSCLC): blood and tissue TMB analysis from Mystic, a phase III study of first-line durvalumab \pm tremelimumab vs chemotherapy. AACR 2019;\# CT074

13. Antonia SJ, Villegas A, Daniel D, Vicente D, Murakami S, Hui R, et al Overall survival with durvalumab after chemoradiotherapy in stage III NSCLC. N Engl J Med. 2018;379(24):2342-50.

14. Gandhi L, Rodríguez-Abreu D, Gadgeel S, Esteban E, Felip E, De Angelis $F$, et al. Pembrolizumab plus chemotherapy in metastatic nonsmall-cell lung cancer. N Engl J Med. 2018;378(22):2078-92.

15. Lu S, Stein JE, Rimm DL, Wang DW, Bell JM, Johnson DB, et al. Comparison of biomarker modalities for predicting response to PD-1/ PD-L1 checkpoint blockade: a systematic review and meta-analysis. JAMA Oncol. 2019. 